\title{
THE LOW FREQUENCY SPACE ARRAY (LFSA)
}

K.W. Weiler ${ }^{1}$ B.K. Dennison ${ }^{2}$ K.J. Johnston ${ }^{1}$, R.S. Simon ${ }^{1}$, J.H. Spencer ${ }^{1}$, W.C. Erickson ${ }^{3}$, M.L. Kaiser ${ }^{4}$, H.V. Cane ${ }^{4}$, M.D. Desch $^{4}$, and L.M. Hammarstrom ${ }^{1}$

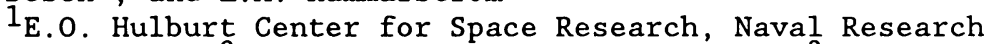
Laboratory, 2Virginia Polytechnic Institute, 3niversity of Maryland, ${ }^{4}$ NASA Goddard Space Flight Center

At the lowest radio frequencies ( $\leq 30 \mathrm{MHz}$ ), the Earth's ionosphere transmits poorly or not at all. This relatively unexplored region of the electromagnetic spectrum is thus an area where high resolution, high sensitivity observations can open a new window for astronomical investigations. Also, extending observations down to very low frequencies brings astronomy to a fundamental physical limit where the Milky Way becomes optically thick over relatively short path lengths due to diffuse free-free absorption.

To obtain data at these frequencies requires the difficulty and expense of placing radio telescopes into Earth orbit, but the scientific rewards of a space mission are likely to be great. Even without considering the serendipitous discoveries which have always accompanied the opening of a new realm of frequency, resolution, or sensitivity in astronomy, a low frequency telescope in space can:

1. map the entire sky, with emphasis on the galactic background non-thermal emission, with high resolution and sensitivity;

2. determine the distribution of galactic diffuse ionized hydrogen by surveying its absorption to discrete background sources;

3. study of the interstellar plasma by investigating the origin, distribution, and magnitude of its scattering and refraction;

4. study individual source spectra for energy production mechanisms and such processes as synchrotron selfabsorption, Razin-Tsytovich effect, HII absorption, inverse Compton scattering, and synchrotron losses;

5. study the origin of the correlation between low frequency steep spectrum clusters of galaxies and their enhanced $\mathrm{x}$-ray emission;

6. search for "fossil" radio components in "radio quiet" objects and extend the counts of sources to the low frequencies where synchrotron lifetimes approach the age of the universe; 
7. image individual sources with high resolution $\left(\sim 10^{\prime}\right.$ at $-1 \mathrm{MHz}$ to $\sim 5$ " at $-30 \mathrm{MHz}$ ) to investigate spectral index changes across source components and to search for extended halos;

8. study the impulsive emission from Jupiter and the Sun and search for similar radiation from other Solar System bodies; and

9. search for the coherent radiation found from Solar System bodies but undetected so far in larger systems.

A scientific justification and technical description has been developed for construction of a Low Frequency Space Array (LFSA) to carry out full sky mapping and individual source imaging between 1.5 and $26.3 \mathrm{MHz}$.
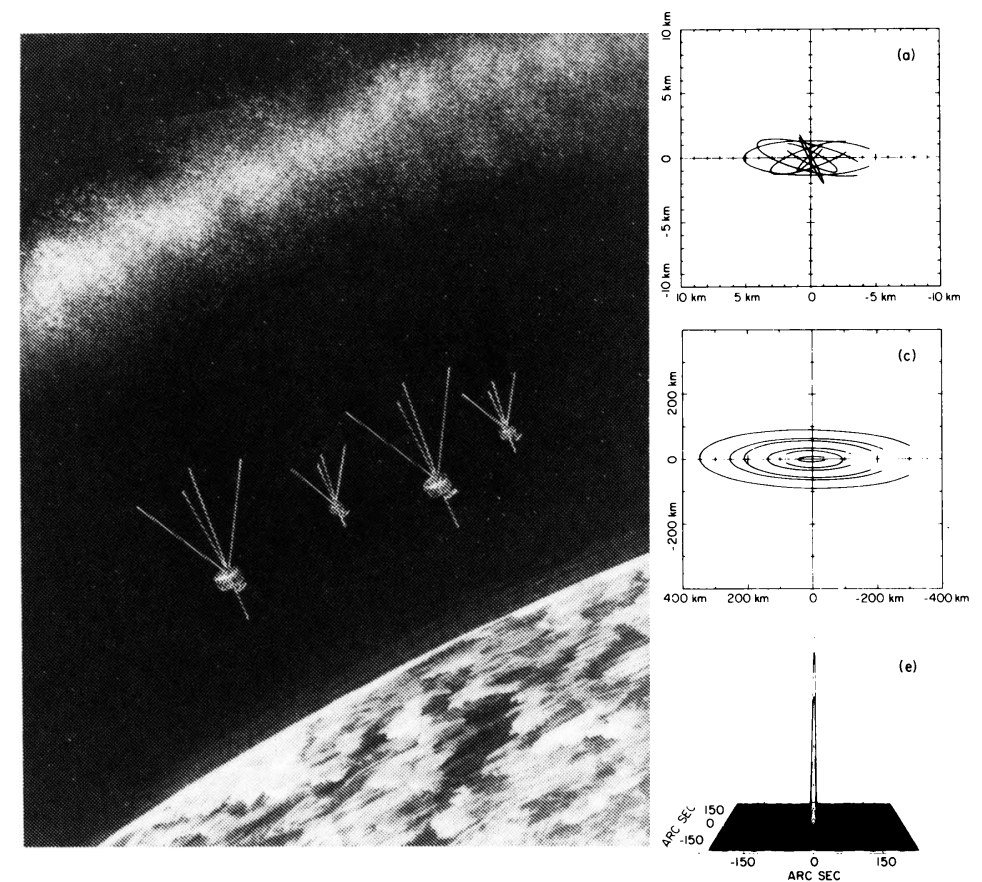
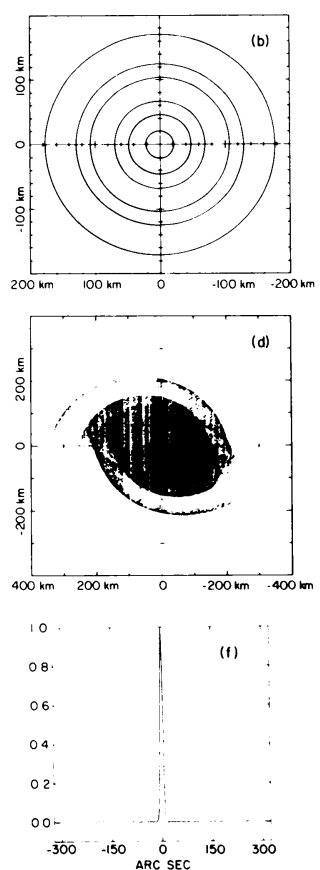

Artist's conception of the Low Frequency Space Array (LFSA) with four array elements.
Aperture plane coverage by a 4 element array for a source at RA $=6^{\mathrm{h}}$, Dec $=45^{\circ}$ for one orbit on (a) Day 1, (b) Day 180, and (c) Day 360. The total coverage during 1 year is given in (d) and the resulting beam pattern in (e). For a cross cut through the beam pattern better illustrating the sidelobe levels see (f). 